\title{
Proteomic characterization of idiopathic pulmonary fibrosis patients: stable versus acute exacerbation
}

\author{
Alfonso Carleo ${ }^{1,2}$, Claudia Landi ${ }^{3}$, Antje Prasse ${ }^{1,2}$, Laura Bergantini ${ }^{4}$, Miriana d'Alessandro ${ }^{4}$, \\ Paolo Cameli $^{4}$, Sabina Janciauskiene ${ }^{2}$, Paola Rottoli ${ }^{4}$, Luca Bini ${ }^{3}$, Elena Bargagli ${ }^{4}$ \\ ${ }^{1}$ Department of Pneumology, Hannover Medical School (MHH), Hannover, Germany; ${ }^{2}$ DZL BREATH, Hannover, \\ Germany; ${ }^{3}$ Functional Proteomic Section, Department of Life Sciences, University of Siena, Italy; ${ }^{4}$ Unit of Respiratory \\ Diseases and Lung Transplantation, Department of Internal and Specialist Medicine, University of Siena, Italy
}

\begin{abstract}
Acute exacerbations (AEs) are among the main causes of death in idiopathic pulmonary fibrosis (IPF) patients. In this study proteomic comparative analysis of bronchoalveolar lavage (BAL) fluid samples was performed in stable IPF patients versus AEs IPF group to identify AE pathogenetic mechanisms and novel potential predictive biomarkers. A functional proteomic analysis of BAL fluid samples from stable and AE-IPF patients was conducted in a population of 27 IPF patients. Fifty-one differentially abundant spots were observed and identified by mass spectrometry.
\end{abstract}

\footnotetext{
Correspondence: Claudia Landi, Department of Life Sciences,

University of Siena,

Via Aldo Moro 2, 53100 Siena, Italy.

Tel. +39.057.234937.

E-mail: landi35@unisi.it

Conflict of interest: The authors have no conflict of interest to declare.

Keywords: Idiopathic pulmonary fibrosis; acute exacerbation; bronchoalveolar lavage; proteomics; protease/antiprotease imbalance.

Contributions: EB, PR, AC, conceived and designed the project; CL, $\mathrm{AC}, \mathrm{LBe}$, designed the experiments; AC, CL, MdA, SJ, performed the experiments; AC, CL, PC, LBi, analyzed the data; AC, CL, EB wrote the manuscript; $\mathrm{EB}, \mathrm{AP}, \mathrm{PR}$, critically reviewed the manuscript; all authors approved the manuscript.
}

Ethics approval and consent to participate: This study received the local Ethical Committee endorsement C.E.A.V.S.E. (code 180712).

Informed consent: Written informed consent was obtained from the patients included in the study.

Received for publication: 10 January 2020

Accepted for publication: 10 March 2020.

${ }^{\circ}$ Copyright: the Author(s), 2020

Licensee PAGEPress, Italy

Monaldi Archives for Chest Disease 2020; 90:1231

doi: 10.4081/monaldi.2020.1231

This article is distributed under the terms of the Creative Commons Attribution Noncommercial License (by-nc 4.0) which permits any noncommercial use, distribution, and reproduction in any medium, provided the original author(s) and source are credited.
Enrichment analysis found proteins of interest involved in the regulation of macrophages and lipid metabolism receptors. In acute exacerbation IPF group, differentially abundant proteins were involved in propagation of the $\beta$-catenin WNT transduction signal, and proteins up-regulated in lung carcinogenesis (IGKC, S100A9, PEDF, IGHG1, ALDOA, A1AT, HPT, CO3 and PIGR) and acute phase proteins involved in protease-antiprotease imbalance (such as A1AT fragments). Dot-blot analysis of A1AT C-36 peptide allowed validating our findings, confirming up-regulation in $\mathrm{AE}$ IPF patients and suggesting its potential pathogenetic role. A crucial role of protease/antiprotease imbalance, clathrin-mediated endocytosis signalling and carcinogenesis emerged in IPF patients developing acute exacerbations.

\section{Introduction}

Idiopathic pulmonary fibrosis (IPF) is one of the most widespread fatal idiopathic interstitial pneumonias of unknown etiology [1] and the prognosis of IPF is extremely poor (median survival 3-5 years). Respiratory failure is the main cause of death, affecting about $80 \%$ of IPF patients [2]. Chronic progressive parenchymal fibrosis, characterized by usual interstitial pneumonia (UIP) pattern with fibroblast foci and "honeycomb" lesions, leads to decay in physiological alveolar structure and decline in respiratory function [2-6]. Diagnosis of IPF is often challenging $[7,8]$ and the clinical course of IPF is variable, unpredictable and irreversible [9]. Some patients have accelerated lung function deterioration with rapid decline and unpredictable acute exacerbations (AEs) that can ensue in $20-55 \%$ of cases $[10,11]$. AEs are considered a major lethal complication of IPF and are associated with high short-term mortality $(50-85 \%$ of deaths occur within weeks of an AE) [11-13]. Miscellaneous risk factors for AE have been proposed, including pulmonary hypertension, gastroesophageal reflux, emphysema, prolonged mechanical ventilation, bronchoscopy and lung biopsy procedures [14]. Unfortunately, there are no well-established international guidelines or standardized diagnostic approaches for AEs of IPF, none are sufficiently sensitive, nor is there any effective standard therapy for AE. Thus, there is an urgent need to find reliable biomarkers of IPF severity for patient stratification. To this purpose, proteomic approach gives a panorama of the complex network of proteins of different origins and functions and their modifications, providing new information about pulmonary microenvironment events for insights into lung physiology and pathophysiology, in addition to suggests potential prognostic, diagnostic and/or theragnostic 
biomarkers. Several proteomic studies performed on bronchoalveolar lavage (BAL) from IPF patients pointing out proteins potentially related to IPF pathogenesis or putative diseases, risk, or stratification biomarkers. Increased profibrotic cytokines and chemokines (like IL-8 and CXCL1) were observed in BAL from IPF patients presenting AE [15], whereas ELANE, KL-6, LDH, SPA, MMP-1 and -7, CCL-18 and -24, LEP, CRP, IL-17, or PDGF were reported to in blood [16-22]. In particular, our group performed a differential proteomic study on BAL fluid in order to extrapolate potential biomarkers of IPF highlighting up-regulation of annexin A2 and peroxiredoxin 1 and the down-regulation of plastin 2. Moreover, the system biology study performed on the differential proteins found, highlighted the major molecular pathways involved such as: "response to hypoxia and oxidative stress" and "iron transport". Interestingly, all mechanisms that we extrapolated from our analysis resulted related with the action of TGF- $\beta$ in fibrosis onset [23]. Since the encouraging results obtained with this research, we were interested in studying potential differential protein patterns able to stratify our IPF samples. In particular, since no reliable biomarkers of AE risk are available in the clinical practice $[13,15,16,23-25]$, we wanted to extrapolate potential differential proteins characterizing AE condition with respect to stable/progressive. In this study we compared for the first time, at the best of our knowledge, BAL samples performed at the moment of diagnosis from patients remained stable/progressive and BAL performed at the moment of AE. The differential proteins found have been subjected to functional analysis to highlight potential molecular ways leading to AE.

\section{Materials and Methods}

\section{Population}

The population consisted of 27 IPF patients (17 males and 10 females; 13 never- and 14 ex-smokers; average age $64 \pm 7$ years). Diagnosis of IPF was performed at Siena Regional ILD Referral Centre and Lung Transplantation Unit according to international ATS/ERS criteria [2]. Diagnosis of AE was based on international criteria, according to Collard et al. [26]. The clinical course of patients had been monitored for at least 36-48 months after the diagnosis. Eighteen IPF patients showed stable disease ( 9 males and 9 females; 8 never- and 10 ex-smokers; average age $66 \pm 6$ years) and have been included in proteomic analysis as "stable group" while 9 patients experienced acute exacerbations without a known cause or agent ( 8 males, 1 female; 4 never- and 5 ex-smokers; AE, average age $60 \pm 8$ years) and were included as "AE group". Bronchoscopy with BAL was performed for diagnostic purposes after written informed consent of IPF patients, at onset of the disease in stable IPF patients and at the moment of clinical-radiological diagnosis of AE before starting high doses of steroids in the group of AE-IPF. At the moment of the bronchoscopy $3 / 18$ stable patients were treated with antifibrotic drugs ( 2 pirfenidone and 1 nintedanib, these patients performed BAL at the starting diagnostic moment) while $8 / 9 \mathrm{AE}$ patients were in treatment with antifibrotic drugs (three with pirfenidone and five with nintedanib). All patients signed the informed consent and this study received the local Ethical Committee endorsement C.E.A.V.S.E. (code number 180712).

The BALF protein abundances of $\alpha$-1-antitrypsin (A1AT) and of its C-terminal fragment (C-36 peptide), corresponding to residues 359-394, were evaluated in an independent patient cohort by Dotblot analysis (Supplementary Material S1). These samples were collected at the Hannover Medical School (MHH, Germany) from
9 IPF patients ( 7 males and 2 females; 4 never- and 5 formersmokers; average age of $73.4 \pm 5.8$ years). Four patients had stable-IPF course ( 3 males, 1 female; 3 ex-, 1 never-smoker, average age of $75 \pm 7.5$ years) and five cases presented AE ( 4 males, 1 female; 2 ex-, 3 never-smokers, average age of $72.2 \pm 4.6$ years).

\section{Sample preparation}

BAL samples were filtered through sterile gauze and BAL cells count and lymphocyte phenotyping was carried out using BD FacsCaliburflow cytometry (BD Biosciences Becton, Dickinson and Company, San Jose, CA, USA). BALF samples were separated and dialyzed as previously reported [23].

\section{D-electrophoresis}

After Bradford assay, lysis buffer (and trace of bromophenol blue) was added until the protein concentrations were adjusted to $60 \mu \mathrm{g}$ in $100 \mu \mathrm{l}$ and $700 \mu \mathrm{g}$ of protein in $200 \mu \mathrm{l}$ of solution for analytical and MS-preparatory 2D-Electophoresis (2DE), respectively. The isoelectric focusing was performed through immobiline polyacrylamide strips with $18 \mathrm{~cm}$ in length and immobilized non-linear $\mathrm{pH}$ gradient 3-10 (GE Healthcare, Uppsala, Sweden). In particular, the strips were rehydrated with a solution containing urea $8 \mathrm{M}, 4 \% \mathrm{w} / \mathrm{v}$ CHAPS, $1 \% \mathrm{w} / \mathrm{v}$ DTE for $12 \mathrm{~h}$ and the samples were loaded by cup-loading method. For isoelectric focusing, the samples were added of $0.2 \%$ and $2 \% \mathrm{v} / \mathrm{v}$ of carrier ampholyte for analytical and MS-preparatory run, respectively. First electrophoretic dimension was carried out using Ettan ${ }^{\mathrm{TM}}$ IPGphor ${ }^{\mathrm{TM}}$ system (Amersham Biosciences, Little Chalfont, UK) at $16^{\circ} \mathrm{C}$, with the following electrical conditions: $0 \mathrm{~V}$ for $1 \mathrm{~h}, 30 \mathrm{~V}$ for $8 \mathrm{hs}, 200 \mathrm{~V}$ for $1 \mathrm{~h}$, from 300 to $3500 \mathrm{~V}$ in $30 \mathrm{~min}, 3500 \mathrm{~V}$ for $3 \mathrm{~h}$, from 3500 to $8000 \mathrm{~V}$ in $30 \mathrm{~min}, 8000 \mathrm{~V}$, for a total of $80,000 \mathrm{Vh}$ at constant temperature of $16^{\circ} \mathrm{C}$. Before the SDS PAGE separation, strips were incubated for 12 min with a buffer containing $6 \mathrm{M}$ urea, $2 \% \mathrm{w} / \mathrm{v}$ Sodium Dodecyl Sulphate (SDS), $2 \% \mathrm{w} / \mathrm{v}$ DTE, $30 \% \mathrm{v} / \mathrm{v}$ glycerol and $0.05 \mathrm{M}$ Tris-HCl pH 6.8 , and, subsequently, with the same solution where DTE was replaced by $2.5 \% \mathrm{w} / \mathrm{v}$ iodoacetamide and a trace of bromophenol blue for further $5 \mathrm{~min}$. The SDS-PAGE run was performed using 9-16\% SDS polyacrylamide linear gradient gels with size of $18 \times 20 \times 1.5 \mathrm{~cm}$ and constant current of $40 \mathrm{~mA} / \mathrm{gel}$ at $9^{\circ} \mathrm{C}$ until the dye front reached the bottom of the gel. The gels for MS-preparatory were stained with SYPRO Ruby (Bio-rad Laboratories, Hercules, CA, USA). Bind-silane ( $\gamma$ methacryloxypropyltrimethoxysilane) (LKBProdukter AB, Brommo, Sweden) was used to attach polyacrylamide gels covalently to a glass surface for those undergoing SYPRO Ruby staining and digitized with Typhoon 9400 laser densitometer (GE Healthcare, Chicago, IL, USA). Ammoniac silver nitrate staining is used to stain the analytical gels that were digitalized thanks to ImageScanner III managed by LabScan 6.0 (GE Healthcare).

\section{D-image and statistical analysis}

Spots were detected and quantified (in term of relative percentage of volume, $\% \mathrm{~V}$ ) in each gel using Image Master Platinum 7.0 software (GE Healthcare). Gels were then grouped in respective class and a reference gel for each class (called Master gel) was selected. Each gel was compared with appropriate Master (Intra-class matching) and consequently the Master gels were compared each other (Inter-class matching). Spots were considered differentially abundant between two conditions when the ratio of the $\% \mathrm{~V}$ means was greater than \pm 2 folds. Student's $t$, WilcoxonMann-Whitney, False Discovery Rate (FDR) tests, unsupervised and supervised Principal Component Analysis, heatmap with tree 
clustering, and general linear regression models were performed by RStudio Desktop 1.1.463 (Integrated Development for RStudio, Inc., Boston, MA, USA, https://www.rstudio.com) in order to validate the statistical significance of comparisons between two classes (Supplementary Materials).

\section{Protein identification}

After excision from MS-preparatory gels by Ettan Spot Picker (GE Healthcare), the spots of interest were destained in $5 \mathrm{mM}$ ammonium bicarbonate and 50\% acetonitrile solution and then completely dehydrated in acetonitrile solution. The gel spots were incubated at $37^{\circ} \mathrm{C}$ overnight in $50 \mathrm{mM}$ ammonium bicarbonate and trypsin solution for protein digestion. Peptide masses were acquired using UltrafleXtreme ${ }^{\mathrm{TM}}$ MALDI-ToF/ToF (Brucher Corp., Billerica, MA, USA), arranging each digested protein solution onto a MALDI support and embedding it with $\alpha$-cyano-4-hydroxycinnamic acid (CHCA), $50 \% \mathrm{v} / \mathrm{v} \mathrm{ACN}$ and $0.5 \% \mathrm{v} / \mathrm{v}$ TFA matrix. Protein identification was carried out by peptide mass fingerprinting (PMF) approach by Mascot online tool (Matrix Science Ltd., London, UK) (Supplementary Materials).

\section{Clustering, pathway and enrichment analysis}

Functional clustering analysis was carried out by DAVID 6.7 (Database for Annotation, Visualization and Integrated Discovery; Frederick, MD, USA; david.abcc.ncifcrf.gov) using Biological Process, Cellular Component, and Molecular Function Gene Ontology (GO) terms together with REACTOME_PATHWAY, and CGAP_SAGE_QUARTILE as database (Supplementary Materials).

\section{Dot-blot analysis}

The BALF protein abundances of $\alpha$-1-antitrypsin (A1AT) and of its C-terminal fragment (C-36 peptide), corresponding to residues 359-394, were also evaluated in the German independent patient cohort by Dot-blot analysis (Supplementary Materials).

\section{Results}

\section{Population}

Table 1 showed the pulmonary function test results, BAL cellular populations and anthropometric data of IPF patient cohort. In the 2-DE population, stable patients were older with higher residual volumes and total lung capacities than AE patients, whereas in the dot-Blot cohort, the only statistical difference concerned the reduction of $\mathrm{FVC} \%$ in $\mathrm{AE}$ cases.

In order to evaluate potentially disturbing effects of other parameters on the protein abundances, general linear regression models were performed where the protein abundance was function of age, gender, batch, or therapy.

The models did not emphasize any particular importance of these parameters (data not shown); the highest significant R.squared value was lower than $\leq 0.3$.

\section{Proteomic analysis}

Eight hundred and fifty spots were detected and matched in BAL from IPF patients by proteomic approach. Comparison of AE and

Table 1. Average values of Pulmonary Function Testing and BAL cell count features of analyzed IPF patient cohorts in 2-DE and Dotblot experiment. The respective standard deviations are reported in brackets.

\begin{tabular}{|c|c|c|c|c|c|c|c|c|}
\hline & Sien & a cohort (2D) & trophore & & & Jannover coh & (Dot-blot & \\
\hline & Prog IPF & AE IPF & p-value & $\mathrm{FC}$ & Prog IPF & AE IPF & p-value & $\mathrm{FC}$ \\
\hline Age (y) & $66.1( \pm 6.3)$ & $59.9( \pm 7.7)$ & $3.25 \mathrm{E}-02$ & 0.91 & $75( \pm 7.5)$ & $72.2( \pm 4.6)$ & 4.13E-01 & 0.96 \\
\hline$\% \mathrm{VC}$ & $76.2( \pm 15)$ & $64.5( \pm 14.4)$ & 2.31E-01 & 0.85 & na & na & na & na \\
\hline$\% \mathrm{FVC}$ & $78.2( \pm 16.7)$ & $68.1( \pm 16.7)$ & 2.78E-01 & 0.87 & $81( \pm 10.7)$ & $53.4( \pm 7.9)$ & $1.95 \mathrm{E}-02$ & 0.66 \\
\hline$\% \mathrm{FEVl}$ & $82.2( \pm 17.4)$ & $74( \pm 17.1)$ & $3.08 \mathrm{E}-01$ & 0.90 & na & na & na & na \\
\hline FEV1NC & $83.9( \pm 4.2)$ & $86.8( \pm 4.6)$ & 1.81E-01 & 1.03 & na & na & na & na \\
\hline VC (ml) & $2281.5( \pm 735.5)$ & $2250( \pm 576.3)$ & $9.72 \mathrm{E}-01$ & 0.99 & na & na & na & na \\
\hline FVC (ml) & $2324.7( \pm 737.4)$ & $2284( \pm 669.2)$ & 6.71E-01 & 0.98 & $2697.5( \pm 724.2)$ & $1910( \pm 516.8)$ & $6.35 \mathrm{E}-02$ & 0.71 \\
\hline RV & $102.9( \pm 11.6)$ & $68.6( \pm 19.4)$ & 1.09E-02 & 0.67 & na & na & na & na \\
\hline TLC & $85.5( \pm 9.4)$ & $65.2( \pm 12.1)$ & 2.64E-02 & 0.76 & $74.25( \pm 18.9)$ & $58( \pm 11)$ & $1.10 \mathrm{E}-01$ & 0.78 \\
\hline DLCO & $46.8( \pm 15.8)$ & $35.1( \pm 10.1)$ & $6.75 \mathrm{E}-02$ & 0.75 & $57.3( \pm 15.3)$ & $45.7( \pm 23)$ & 4.00E-01 & 0.80 \\
\hline $\mathrm{KCO}$ & $71.7( \pm 21.4)$ & $69.7( \pm 17.9)$ & $8.05 \mathrm{E}-01$ & 0.97 & na & na & na & na \\
\hline \% Macrophages & $66.6( \pm 21.9)$ & $69.7( \pm 13.9)$ & 9.38E-01 & 1.05 & $83( \pm 14.1)$ & $81.2( \pm 6.8)$ & 3.83E-01 & 0.98 \\
\hline \% Lymphocytes & $16.5( \pm 14.2)$ & $10.4( \pm 4.6)$ & 5.71E-01 & 0.63 & $5.5( \pm 1.3)$ & $11.8( \pm 6.1)$ & $1.74 \mathrm{E}-01$ & 2.15 \\
\hline \% Neutrophils & $7.3( \pm 9)$ & $9.1( \pm 6.4)$ & 4.54E-01 & 1.25 & $7.8( \pm 9.6)$ & $5.2( \pm 3.4)$ & $1.00 \mathrm{E}+00$ & 0.67 \\
\hline \% Eosinophils & $9.6( \pm 18.9)$ & $7.4( \pm 7.1)$ & 8.36E-01 & 0.77 & $2.3( \pm 2.3)$ & $1.6( \pm 1.1)$ & 7.97E-01 & 0.70 \\
\hline \% CD3+ & $76.5( \pm 12.3)$ & $77.9( \pm 9.3)$ & 7.46E-01 & 1.02 & na & na & na & na \\
\hline$\% \mathrm{CD} 4+$ & $52.8( \pm 14.1)$ & $44.8( \pm 18)$ & 2.87E-01 & 0.85 & na & na & na & na \\
\hline$\%$ CD8+ & $22.9( \pm 8.9)$ & $33.1( \pm 14.4)$ & $1.06 \mathrm{E}-01$ & 1.45 & na & na & na & na \\
\hline$\% \mathrm{CD} 19+$ & $1.4( \pm 1.5)$ & $1.2( \pm 1)$ & 9.24E-01 & 0.86 & na & na & na & na \\
\hline CD4+/CD8+ & $3( \pm 2.3)$ & $1.8( \pm 1.5)$ & 6.62E-02 & 0.60 & na & na & na & na \\
\hline
\end{tabular}

FC, fold change (ratio AE/Prog means); na, data not available; VC, vital capacity; FEVl, forced expiratory volume in 1 s; FVC, forced vital capacity; RV, residual volume; TLC, total lung capacity; DLCO, diffusing capacity or transfer factor of the lung for carbon monoxide; $\mathrm{KCO}$, transfer coefficient of the lung for carbon monoxide. 
stable IPF groups revealed 51 differentially abundant spots, all efficiently identified by mass spectrometry (Table 2). These differentially abundant spots had a $\% \mathrm{~V}$ mean ratio of at least 2-fold between the two groups $(p<0.05) ; 38 / 51$ spots were increased in the AE group than stable IPF group, whereas 13 spots were higher in stable IPF patients with respect to the AE group (Table 2). Most of the 51 identified proteins were isoforms/proteoforms (same protein with different post translational modifications) and have the same protein name. For this reason, we observed 18 unique proteins. In particular, Annexin A1 (ANXA1), fructose-bisphosphate aldolase A (ALDOA), leucine-rich $\alpha$-2-glycoprotein (LRG1), Ig $\mathrm{k}$ chain (IGKC), protein S100A8, protein S100A9, alpha 1-antitrypsin (A1AT), A1AT C-term fragment, one proteoform of albumin (ALBU), haptoglobin (HPT), polymeric immunoglobulin receptor (PIGR), serum transferrin (TRFE), Ig $\gamma$ chain (IGHG1) were upregulated in AE-IPF. On the other hand, complement C3 C-term, pigment epithelium-derived factor (PEDF), selenium-binding protein 1 (SBP1) and $\alpha$-1B-glycoprotein (A1BG) were down-regulated in AE-IPF. Tables S-1 and 2 showed the results of spot identification and spot statistical values, respectively. Figure 1 reported the abundance of these proteins in the two groups (in the heat map proteins upregulated were in green and down-regulated in red).

\section{Principal component analysis (PCA)}

Figure 2 reported the different spatial rotations of the PCA 3D plot. The eigenvalue percentage significance of the first three PCs was $72.3 \%$. The two groups resulted separated along the first PC. A high homogeneity was observed for AE IPF group and not for stable IPF patients (Figure 2). Intraclass dispersion occurred mainly along the first and third PCs for the stable and AE groups. The stable group core seemed located at positive $\mathrm{X}$ - and negative $\mathrm{z}$-values (first Cartesian octant), whereas that of the AE group spread over negative $\mathrm{x}$ - and z-values (second and fourth octants).

\section{Clustering, enrichment and pathway analysis}

DAVID functional clustering (Table S-2) demonstrated 12 significant GO terms for biological process, five for cellular component, four for molecular function, two for pathway, and two for tissue expression. Ingenuity analysis, that integrated experimentally observed proteins of interest into a network showed a network of 109

Table 2. Significant differentially abundant protein spots observed in 2-DE. The mean (and standard deviation) of the higher abundant condition is emphasized in bold.

\begin{tabular}{|c|c|c|c|c|c|c|c|c|}
\hline \# & Symbol & Protein name & $\mathbf{A E}$ & Stable & $\begin{array}{c}\mathrm{p} \text {-value } \\
\mathrm{t}\end{array}$ & $\begin{array}{l}\text { FDR } \\
\text { WMW }\end{array}$ & $\log 2 \mathrm{FC}$ & \\
\hline 1 & LRG1 & Leucine-rich alpha-2-glycoprotein & $0.299( \pm 0.024)$ & $0.009( \pm 0.015)$ & 5.12E-03 & $1.54 \mathrm{E}-02$ & $1.54 \mathrm{E}-03$ & 5.02 \\
\hline 2 & IGHG1 & Ig $\gamma$-1 chain C region & $0.013( \pm 0.014)$ & $0.001( \pm 0.003)$ & 2.91E-03 & 4.37E-03 & $2.20 \mathrm{E}-03$ & 3.66 \\
\hline 3 & ALBU & Serum albumin & $0.089( \pm 0.061)$ & $0.009( \pm 0.019)$ & $4.28 \mathrm{E}-05$ & 8.05E-04 & 4.02E-04 & 3.34 \\
\hline 4 & HPT & Haptoglobin & $0.192( \pm 0.139)$ & $0.029( \pm 0.034)$ & $1.24 \mathrm{E}-04$ & $1.35 \mathrm{E}-03$ & 6.77E-04 & 2.73 \\
\hline 5 & IGHG1 & Ig $\gamma$-1 chain $\mathrm{C}$ region & $0.192( \pm 0.139)$ & $0.029( \pm 0.034)$ & 1.23E-04 & $1.62 \mathrm{E}-03$ & 8.22E-04 & 2.72 \\
\hline 6 & ALBU (C-term) & Serum albumin (C-term) & $0.017( \pm 0.016)$ & $0.003( \pm 0.006)$ & $3.87 \mathrm{E}-03$ & $5.55 \mathrm{E}-03$ & $2.78 \mathrm{E}-03$ & 2.6 \\
\hline 7 & ANXAl & Annexin Al & $0.006( \pm 0.006)$ & $0.001( \pm 0.003)$ & 1.56E-02 & 3.72E-02 & 1.91E-02 & 2.4 \\
\hline 8 & ALBU (C-term) & Serum albumin (C-term) & $0.014( \pm 0.017)$ & $0.003( \pm 0.005)$ & $1.80 \mathrm{E}-02$ & $4.62 \mathrm{E}-02$ & 2.39E-02 & 2.38 \\
\hline 9 & AlAT (C-term) & $\alpha$-1-antitrypsin (C-term) & $0.011( \pm 0.015)$ & $0.002( \pm 0.006)$ & 4.66E-02 & 3.03E-02 & $1.62 \mathrm{E}-02$ & 2.35 \\
\hline 10 & ALBU & Serum albumin & $0.032( \pm 0.025)$ & $0.007( \pm 0.013)$ & 3.35E-03 & 9.19E-03 & 4.65E-03 & 2.14 \\
\hline 11 & ALBU (C-term) & Serum albumin (C-term) & $0.013( \pm 0.008)$ & $0.003( \pm 0.008)$ & 1.10E-02 & 2.25E-03 & 1.13E-03 & 1.97 \\
\hline 12 & ALBU (C-term) & Serum albumin (C-term) & $0.019( \pm 0.018)$ & $0.005( \pm 0.011)$ & 2.32E-02 & $6.55 \mathrm{E}-03$ & $3.32 \mathrm{E}-03$ & 1.83 \\
\hline 13 & ALBU (C-term) & Serum albumin (C-term) & $0.02( \pm 0.011)$ & $0.006( \pm 0.007)$ & 6.94E-04 & $3.40 \mathrm{E}-03$ & 1.73E-03 & 1.81 \\
\hline 14 & S100A9 & Protein S100-A9 & $0.014( \pm 0.013)$ & $0.004( \pm 0.01)$ & 3.79E-02 & $6.62 \mathrm{E}-03$ & 3.38E-03 & 1.8 \\
\hline 15 & AlAT & $\alpha$-1-antitrypsin & $0.091( \pm 0.086)$ & $0.027( \pm 0.022)$ & 8.00E-03 & 3.07E-02 & $1.56 \mathrm{E}-02$ & 1.77 \\
\hline 16 & ALBU & Serum albumin & $0.131( \pm 0.118)$ & $0.04( \pm 0.044)$ & $9.72 \mathrm{E}-03$ & 1.15E-02 & $5.97 \mathrm{E}-03$ & 1.73 \\
\hline 17 & IGHGl & Ig $\gamma$-1 chain $\mathrm{C}$ region & $0.131( \pm 0.118)$ & $0.04( \pm 0.045)$ & $9.92 \mathrm{E}-03$ & 1.15E-02 & 5.93E-03 & 1.73 \\
\hline 18 & IGKC & Ig $\kappa$ chain $\mathrm{C}$ region & $0.063( \pm 0.049)$ & $0.019( \pm 0.029)$ & $9.96 \mathrm{E}-03$ & $1.54 \mathrm{E}-02$ & 7.88E-03 & 1.72 \\
\hline 19 & S100A9 & Protein S100-A9 & $0.016( \pm 0.014)$ & $0.005( \pm 0.009)$ & 2.02E-02 & 2.76E-02 & $1.46 \mathrm{E}-02$ & 1.66 \\
\hline 20 & IGHG1 & Ig $\gamma$-1 chain $C$ region & $0.111( \pm 0.082)$ & $0.035( \pm 0.049)$ & $8.28 \mathrm{E}-03$ & 7.39E-03 & $3.70 \mathrm{E}-03$ & 1.66 \\
\hline 21 & ALDOA & Fructose-bisphosphate aldolase A & $0.03( \pm 0.029)$ & $0.01( \pm 0.014)$ & 3.19E-02 & 1.41E-02 & 7.33E-03 & 1.59 \\
\hline 22 & IGHGI & Ig $\gamma$-1 chain $C$ region & $0.11( \pm 0.081)$ & $0.037( \pm 0.043)$ & 6.79E-03 & $3.26 \mathrm{E}-02$ & $1.64 \mathrm{E}-02$ & 1.58 \\
\hline 23 & ALBU (N-term) & Serum albumin (N-term) & $0.044( \pm 0.034)$ & $0.015( \pm 0.025)$ & 2.49E-02 & 1.66E-02 & 8.57E-03 & 1.52 \\
\hline 24 & IGHGl & Ig $\gamma$-1 chain $C$ region & $0.02( \pm 0.017)$ & $0.007( \pm 0.012)$ & $3.92 \mathrm{E}-02$ & $1.05 \mathrm{E}-02$ & $5.36 \mathrm{E}-03$ & 1.51 \\
\hline 25 & IGHG1 & Ig $\gamma-1$ chain $C$ region & $0.132( \pm 0.104)$ & $0.046( \pm 0.058)$ & $1.44 \mathrm{E}-02$ & $3.62 \mathrm{E}-02$ & $1.92 \mathrm{E}-02$ & 1.51 \\
\hline 26 & ALBU & Serum albumin & $0.044( \pm 0.033)$ & $0.016( \pm 0.023)$ & 1.77E-02 & $6.25 \mathrm{E}-03$ & $3.16 \mathrm{E}-03$ & 1.51 \\
\hline 27 & ALBU & Serum albumin & $0.061( \pm 0.039)$ & $0.022( \pm 0.026)$ & $6.23 \mathrm{E}-03$ & $1.13 \mathrm{E}-02$ & $5.68 \mathrm{E}-03$ & 1.49 \\
\hline
\end{tabular}


nodes connected by 679 interactions (Figure 3). In this network IL-6, APOA1 and VEGFA were the main theoretical central hubs, whereas C3, ALB, S100A9 and S100A8 were the proteins with most interactions. Canonical pathways were reported in Table 3 together with "disease and biofunction" and "tox list" results. Interestingly the most relevant pathogenetic pathways identified with this approach were "acute phase response signaling", "atherosclerosis/lipid signaling", "IL-12 signaling and production in macrophages", "clathrin-mediated endocytosis signaling" and "production of nitric oxide and reactive oxygen species in macrophages".

\section{Dot blot analysis}

In order to verify the result of higher A1AT and C-36 peptide levels in BAL samples from AE IPF group with respect to stable group, dot-plot analysis was performed in an independent cohort of validation (Figure 4). The general trends of higher A1AT and C-36 peptide levels in the AE group with respect to the stable group were confirmed. Only one stable patient showed similar up-regulation of A1AT and one AE patient had no detectable C-36 concentration.

\section{Discussion}

Biomarker discovery is a recent topic of interest in ILD and in particular in IPF. In the literature, many molecules have been proposed as potential biomarkers of this disease, however no single protein can be used in the clinical practice for prognostic reasons. Cytokines, CC-chemokines, and other macrophageproduced mediators resulted the most promising prognostic biomarkers of IPF and our comparative proteomic analysis of BAL confirmed a central role of macrophages and their activation receptors (such as LXR and FXR) in IPF, particularly in AE development. The overall biological dissimilarity of the AE and stable IPF groups was demonstrated by 51 differentially abundant spots, the heatmap and PCA results. Interestingly in BAL from AE IPF patients, proteins differentially expressed included: acutephase proteins involved in response signaling, proteins involved in clathrin-mediated endocytosis signaling and lung carcinogenesis. In our study IPF pathogenesis network revealed APOA1, VEGFA, and IL- 6 as the three main hypothetical central

Table 2. Continued from previous page.

\begin{tabular}{|c|c|c|c|c|c|c|c|c|}
\hline$\#$ & Symbol & Protein name & $\mathrm{AE}$ & Stable & $\begin{array}{c}p \text {-value } \\
t\end{array}$ & $\begin{array}{l}\text { FDR } \\
\text { WMW }\end{array}$ & $\log 2 \mathrm{FC}$ & \\
\hline 28 & IGHGI & Ig $\gamma$-1 chain $\mathrm{C}$ region & $0.187( \pm 0.13)$ & $0.067( \pm 0.065)$ & $4.86 \mathrm{E}-03$ & $2.06 \mathrm{E}-02$ & $1.03 \mathrm{E}-02$ & 1.49 \\
\hline 29 & ALBU & Serum albumin & $0.056( \pm 0.033)$ & $0.02( \pm 0.018)$ & 1.73E-03 & 7.30E-03 & $3.66 \mathrm{E}-03$ & 1.47 \\
\hline 30 & IGHG1 & Ig $\gamma$-1 chain C region & $0.131( \pm 0.091)$ & $0.05( \pm 0.046)$ & $6.66 \mathrm{E}-03$ & $4.99 \mathrm{E}-02$ & $2.69 \mathrm{E}-02$ & 1.4 \\
\hline 31 & AlAT & $\alpha$-1-antitrypsin & $0.246( \pm 0.147)$ & $0.096( \pm 0.1)$ & 6.14E-03 & $6.13 \mathrm{E}-03$ & $3.08 \mathrm{E}-03$ & 1.36 \\
\hline 32 & HPT & Haptoglobin & $0.246( \pm 0.147)$ & $0.096( \pm 0.1)$ & $6.16 \mathrm{E}-03$ & $6.13 \mathrm{E}-03$ & $3.09 \mathrm{E}-03$ & 1.36 \\
\hline 33 & TRFE & Serotransferrin & $0.091( \pm 0.059)$ & $0.037( \pm 0.047)$ & $1.95 \mathrm{E}-02$ & $1.59 \mathrm{E}-02$ & 8.31E-03 & 1.31 \\
\hline 34 & ALBU & Serum albumin & $0.074( \pm 0.063)$ & $0.03( \pm 0.034)$ & $3.27 \mathrm{E}-02$ & 2.93E-02 & $1.49 \mathrm{E}-02$ & 1.3 \\
\hline 35 & IGHAl & Ig $\alpha-1$ chain $C$ region & $0.089( \pm 0.058)$ & $0.037( \pm 0.054)$ & $3.59 \mathrm{E}-02$ & $1.43 \mathrm{E}-02$ & 7.48E-03 & 1.27 \\
\hline 36 & AlAT & $\alpha$-1-antitrypsin & $0.115( \pm 0.089)$ & $0.049( \pm 0.043)$ & $1.77 \mathrm{E}-02$ & $7.48 \mathrm{E}-03$ & $3.77 \mathrm{E}-03$ & 1.25 \\
\hline 37 & PIGR & Polymeric immunoglobulin receptor & $0.113( \pm 0.096)$ & $0.049( \pm 0.045)$ & 3.09E-02 & $3.05 \mathrm{E}-02$ & $1.54 \mathrm{E}-02$ & 1.21 \\
\hline 38 & S100A8 & Protein S100-A8 & $0.065( \pm 0.043)$ & $0.03( \pm 0.033)$ & $3.38 \mathrm{E}-02$ & 2.04E-02 & $1.04 \mathrm{E}-02$ & 1.12 \\
\hline 39 & SBP1 & Selenium-binding protein 1 & $0.134( \pm 0.116)$ & $0.325( \pm 0.2)$ & $1.77 \mathrm{E}-02$ & $1.68 \mathrm{E}-02$ & 1.63E-02 & -1.27 \\
\hline 40 & ALBU & Serum albumin & $0.135( \pm 0.116)$ & $0.326( \pm 0.201)$ & $1.78 \mathrm{E}-02$ & $1.68 \mathrm{E}-02$ & $1.61 \mathrm{E}-02$ & -1.27 \\
\hline 41 & AlAT & $\alpha$-1-antitrypsin & $0.154( \pm 0.104)$ & $0.386( \pm 0.199)$ & 4.06E-03 & 6.41E-03 & $6.04 \mathrm{E}-03$ & -1.33 \\
\hline 42 & CERU & Ceruloplasmin & $0.024( \pm 0.025)$ & $0.062( \pm 0.04)$ & 1.79E-02 & $1.00 \mathrm{E}-02$ & $9.92 \mathrm{E}-03$ & -1.39 \\
\hline 43 & TRFE & Serotransferrin & $0.177( \pm 0.106)$ & $0.474( \pm 0.298)$ & $1.00 \mathrm{E}-02$ & $7.48 \mathrm{E}-03$ & $6.99 \mathrm{E}-03$ & -1.42 \\
\hline 44 & PIGR & Polymeric immunoglobulin receptor & $0.03( \pm 0.034)$ & $0.083( \pm 0.069)$ & 4.69E-02 & $3.95 \mathrm{E}-02$ & $3.48 \mathrm{E}-02$ & -1.46 \\
\hline 45 & AlBG & $\alpha$-1B-glycoprotein & $0.022( \pm 0.013)$ & $0.063( \pm 0.038)$ & $5.68 \mathrm{E}-03$ & $1.30 \mathrm{E}-03$ & $1.30 \mathrm{E}-03$ & -1.51 \\
\hline 46 & PEDF & Pigment epithelium-derived factor & $0.009( \pm 0.013)$ & $0.042( \pm 0.037)$ & 1.83E-02 & 2.42E-02 & 2.31E-02 & -2.29 \\
\hline 47 & C3 (C-term) & Complement C3 (C-term) & $0.009( \pm 0.009)$ & $0.074( \pm 0.069)$ & 1.13E-02 & $3.17 \mathrm{E}-02$ & 2.99E-02 & -3.12 \\
\hline 48 & ALBU & Serum albumin & $0( \pm 0)$ & $0.02( \pm 0.023)$ & 2.01E-02 & $7.56 \mathrm{E}-03$ & 7.11E-03 & \\
\hline \multicolumn{9}{|c|}{ Qualitative } \\
\hline \multicolumn{6}{|c|}{ Qualitative } & & & \\
\hline & ALBU (C-term) & Serum albumin (C-term) & $0( \pm 0)$ & $0.013( \pm 0.012)$ & 6.67E-03 & $7.56 \mathrm{E}-03$ & 7.42E-03 & \\
\hline \multicolumn{9}{|c|}{ Qualitative } \\
\hline $\begin{array}{l}51 \\
Q\end{array}$ & tive ${ }^{\text {ALBU }}$ & Serum albumin & $0( \pm 0)$ & $0.032( \pm 0.042)$ & $3.74 \mathrm{E}-02$ & $2.18 \mathrm{E}-02$ & 2.14E-02 & \\
\hline
\end{tabular}

t, Student's $t$-test p-value; WMW, Wilcoxon-Mann-Whitney test p-value; $\log _{2} \mathrm{FC}, \log _{2}$ of fold change AE/stable. 
hubs. Intriguing pathogenetic pathways in AE IPF patients were demonstrated, including: acute phase response signaling, clathrinmediated endocytosis signaling, atherosclerosis signaling, IL-12 signaling and production in macrophages, production of nitric oxide and reactive oxygen species in macrophages. Acute phase response signaling resulted over-expressed in AE samples as a consequence of the up-regulation of TRFE, HPT, C3, CERU, PEDF, A1AT and ALBU. The observed C- terminus of A1AT was highly abundant in AE group and it may be the C36 peptide (residues 359-394) reported to promote inflammation by activation of NF- $\kappa B$, Toll-like receptor 4 and the MAPK pathway [27,28]. Dot-blot analysis suggested an overall increase in C-36 peptide in the AE group with respect to stable IPF patients, indicating this peptide as a possible predictive biomarker of AEs worth of further evaluation. On the other hand, we found down-regulation of PEDF, an important angiostatic and anti-angiogenic glycoprotein, in the AE group. This glycoprotein is involved in collagen binding, wound healing resolution, lung fibroblast chemotaxis, inhibition of fibroblast proliferation and activation of apoptosis through Fas, $\mathrm{NF}-\kappa \mathrm{B}$ and caspase signaling in endothelial cells [17,29]. PEDF is suggested to have antifibrotic effects inhibiting canonical $\mathrm{Wnt} / \beta$ - catenin signaling and expression of different fibrotic genes (through induction of PPAR $\gamma$ signaling), including TGF- $\beta 1$ [18]. Interestingly, the best-known function of PEDF is inhibition of VEGFA, one of our hypothetical central hubs [19] and interesting because its receptor VEGFR is a target of nintedanib [20]. Clathrin-mediated endocytosis signaling, suggested by dysregulation of S100A8, TRFE, A1AT and ALBU, is reported to be important in signal propagation of $\beta$-catenin-dependent WNT signal transduction, fundamental for development, tissue homeostasis and implicated in IPF [21]. It is also reported to be vital in maintaining embryonic stem cell pluripotency [22], a mechanism involved in IPF. Ingenuity analysis likewise revealed the importance of atherosclerosis signaling in the AE group on the basis of dysregulated proteins, such as S100A8, TRFE, A1AT and ALBU. These findings were in line with those of Landi et al. and
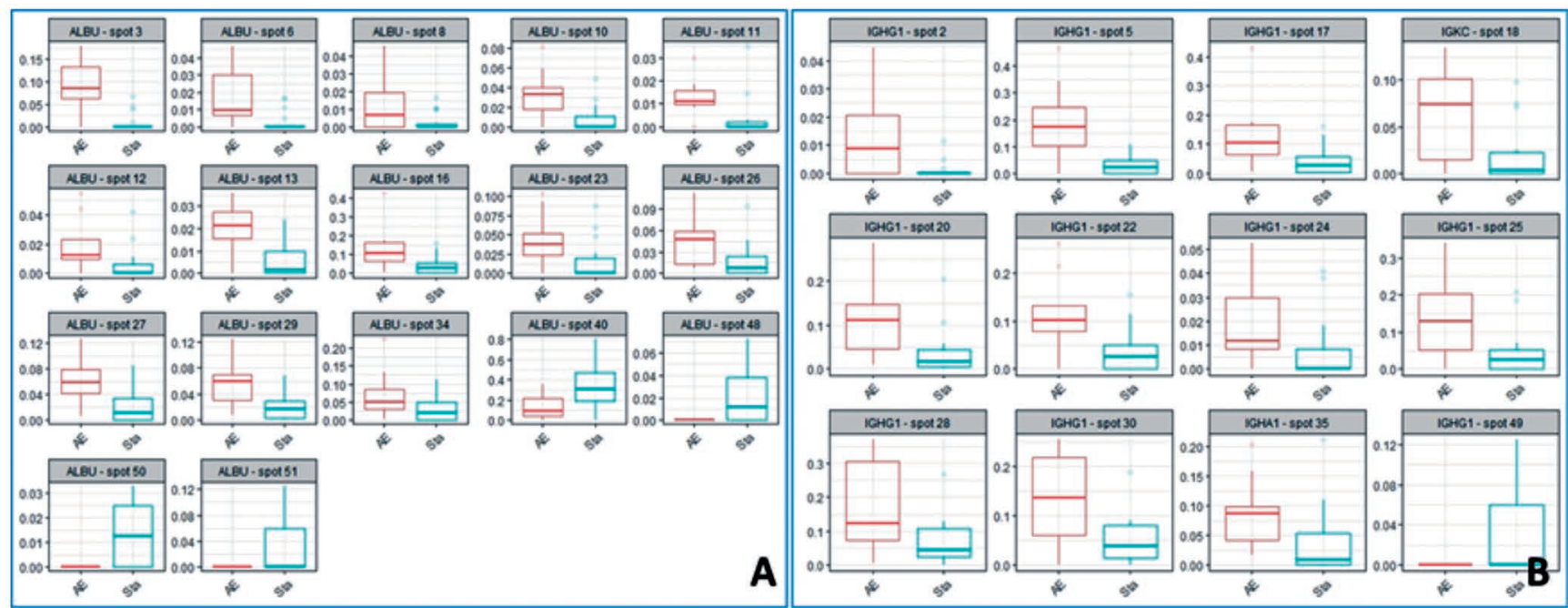

A
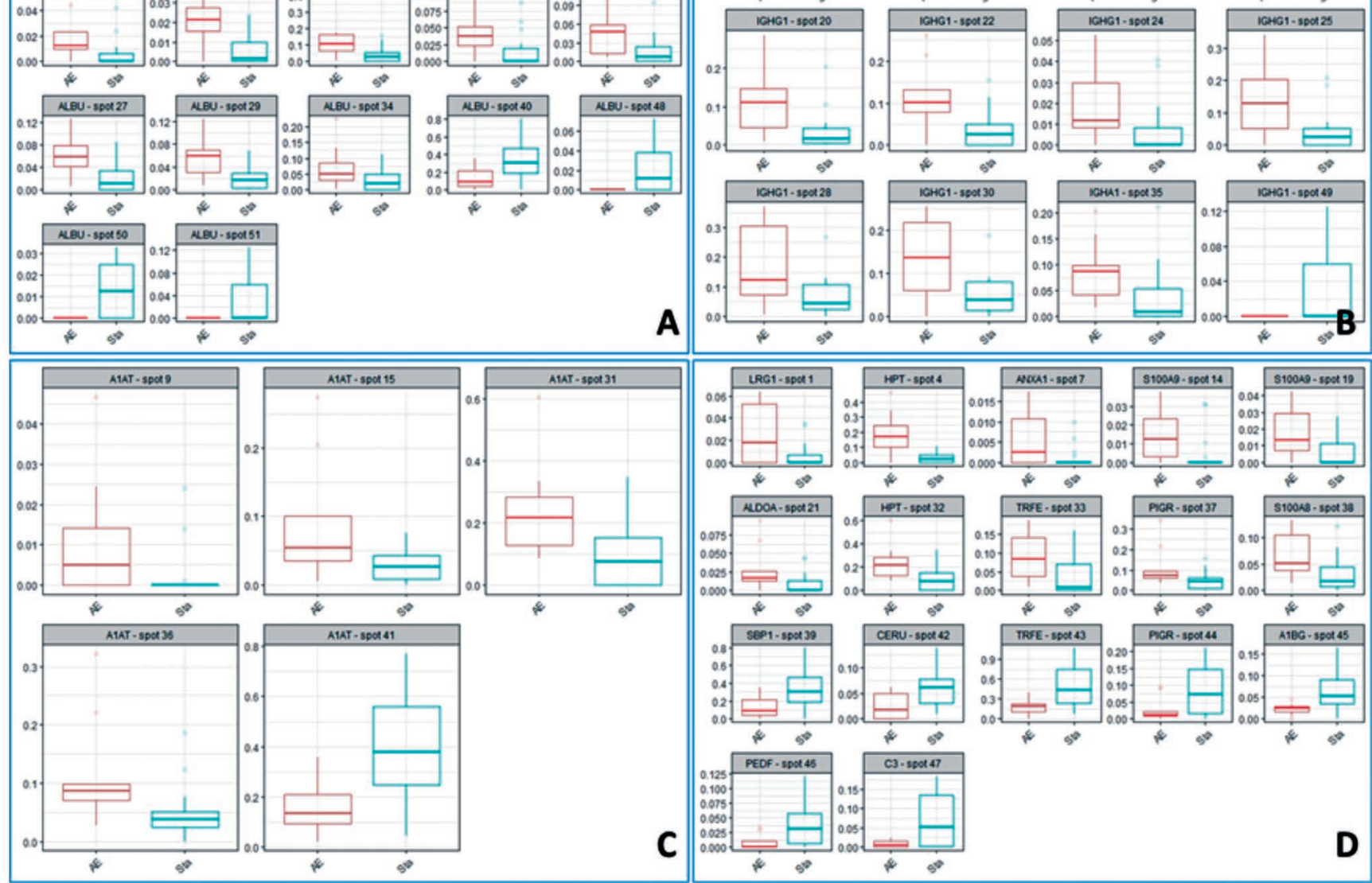

Figure 1. Box plots of the 51 differentially abundant spots between AE (red) and Stable (cyan) IPF. The panels A, B, and C showed the spots corresponding serum albumin, immunoglobulins and A1AT, respectively, whereas panel D reported the other spots of interest. 
Thomas et al. [30-32]. These authors found that the formation of foamy macrophages by sequestration of modified extracellular lipids is a key event in atherosclerosis through over-expression of genes related to fibrosis [32,33]. S100A8, A1AT and ALBU dysregulation also suggests "IL-12 signaling and production in macrophages" and "production of nitric oxide and reactive oxygen species in macrophages", stressing the importance of macrophages and their fine regulation not only in the onset of fibrosis, but also in the evolution of IPF. Finally a relevant result of functional clustering analysis was related to "poorly differentiated lung adenocarcinoma", suggesting that several differentially observed proteins (such as IGKC, S100A9, PEDF, IGHG1, ALDOA, A1AT, HPT, CO3 and PIGR), could be involved in lung carcinogenesis as well as in IPF patients developing AEs [34,35]. The concurrent up-regulation of ALDOA, ANXA1, S100A8, S100A9 and LRG1 and reduced levels of SBP1 and PEDF observed in the AE group in the present study suggest that tumor-like and/or EMT processes in the early stages of IPF may be a pathogenetic mechanism that leads to AEs [36,37].

In conclusion, this study firstly compares proteomic differential pathways of stable IPF patients than AE IPF patients identifying several differential expressed proteins potentially involved in $\mathrm{AE}$ development and many pathogenetic pathways (such as clathrinmediated endocytosis signaling and lung carcinogenesis) to be further validated.

\section{Limitations}

Even if the number of analyzed samples is higher for a 2-DE experiment, the clinical record size is quite low. For these reasons, all the differentially abundant proteins will be further analyzed in larger cohort using different approach such an ELISA test and/or

Table 3. Ingenuity functional clustering analysis.

\begin{tabular}{|c|c|c|c|}
\hline & Category & p-value & Molecules \\
\hline Canonical pathways & $\begin{array}{l}\text { LXR/RXR activation } \\
\text { Acute phase response signaling } \\
\text { FXR/RXR Activation } \\
\text { Clathrin-mediated Endocytosis Signaling } \\
\text { Atherosclerosis signaling } \\
\text { IL-12 signaling and production in macrophages } \\
\text { Production of nitric oxide and reactive oxygen } \\
\text { species in macrophages }\end{array}$ & $\begin{array}{l}1.63 \mathrm{E}-11 \\
2.65 \mathrm{E}-10 \\
1.66 \mathrm{E}-09 \\
4.42 \mathrm{E}-05 \\
2.86 \mathrm{E}-04 \\
4.21 \mathrm{E}-04 \\
1.17 \mathrm{E}-03\end{array}$ & $\begin{array}{l}\text { S100A8, TF, C3, SERPINF1, SERPINA1, A1BG, ALB } \\
\text { TF, HP, C3, CP, SERPINF1, SERPINA1, ALB } \\
\text { TF, C3, SERPINF1, SERPINA1, A1BG, ALB } \\
\text { S100A8, TF, SERPINA1, ALB } \\
\text { S100A8, SERPINA1, ALB } \\
\text { S100A8, SERPINA1, ALB } \\
\text { S100A8, SERPINA1, ALB }\end{array}$ \\
\hline Diseases and biofunctions & $\begin{array}{l}\text { Cellular movement } \\
\text { Hematological system development and function } \\
\text { Immune cell trafficking } \\
\text { Inflammatory response } \\
\text { Cell-to-cell signaling and interaction } \\
\text { Connective tissue disorders } \\
\text { Inflammatory disease } \\
\text { Organismal injury and abnormalities } \\
\text { Skeletal and muscular disorders } \\
\text { Cellular compromise } \\
\text { Immunological disease } \\
\text { Cell signaling } \\
\text { Cellular function and maintenance } \\
\text { Molecular transport } \\
\text { Vitamin and mineral metabolism } \\
\text { Lipid metabolism } \\
\text { Small molecule biochemistry } \\
\text { Infectious diseases } \\
\text { Respiratory disease } \\
\text { Cardiovascular system development and function } \\
\text { Cellular development } \\
\text { Cellular growth and proliferation } \\
\text { Organismal development } \\
\text { Tissue development } \\
\text { Cell death and survival } \\
\text { Lymphoid tissue structure and development }\end{array}$ & $\begin{array}{l}6.63 \mathrm{E}-07 \\
1.99 \mathrm{E}-06 \\
1.99 \mathrm{E}-06 \\
1.99 \mathrm{E}-06 \\
1.99 \mathrm{E}-06 \\
8.99 \mathrm{E}-06 \\
2.14 \mathrm{E}-05 \\
2.16 \mathrm{E}-05 \\
2.16 \mathrm{E}-05 \\
2.16 \mathrm{E}-05 \\
2.16 \mathrm{E}-05 \\
1.04 \mathrm{E}-04 \\
1.04 \mathrm{E}-04 \\
2.86 \mathrm{E}-04 \\
2.86 \mathrm{E}-04 \\
9.35 \mathrm{E}-04 \\
9.35 \mathrm{E}-04 \\
9.35 \mathrm{E}-04 \\
9.35 \mathrm{E}-04 \\
9.35 \mathrm{E}-04 \\
1.15 \mathrm{E}-03 \\
4.13 \mathrm{E}-03\end{array}$ & $\begin{array}{l}\text { ALB, C3, S100A9, ANXA1, SERPINF1, S100A8, SERPINA1, PIGR } \\
\text { ALB, C3, S100A9, ANXA1, S100A8, SERPINA1, IGHA1, IGHG1, PIGR } \\
\text { ALB, C3, S100A9, ANXA1, S100A8, SERPINA1, IGHG1, PIGR } \\
\text { HP, ALB, C3, S100A9, IGKC, ANXA1, ALDOA, SERPINA1, S100A8, } \\
\text { IGHG1, IGHA1, PIGR } \\
\text { C3, S100A9, ANXA1, SERPINA1, S100A8, IGHG1, IGHA1, PIGR } \\
\text { HP, C3, S100A9, IGKC, ALDOA, S100A8 } \\
\text { HP, C3, S100A9, IGKC, ALDOA, SERPINA1, S100A8 } \\
\text { HP, C3, S100A9, ANXA1, IGKC, ALDOA, SERPINA1, S100A8 } \\
\text { HP, C3, S100A9, IGKC, ALDOA, S100A8 } \\
\text { ALB, C3, SERPINA1, IGHA1 } \\
\text { HP, C3, S100A9, ALDOA, S100A8 } \\
\text { C3, S100A9, ANXA1, SERPINA1, S100A8 } \\
\text { C3, ANXA1, SERPINF1, SERPINA1, IGHA1, IGHG1 } \\
\text { C3, S100A9, ANXA1, SERPINA1, S100A8 } \\
\text { C3, S100A9, ANXA1, S100A8, SERPINA1 } \\
\text { C3, ANXA1, SERPINA1 } \\
\text { C3, ANXA1, SERPINA1 } \\
\text { HP, S100A9, SERPINA1 } \\
\text { HP, S100A9, ANXA1, SERPINA1 } \\
\text { C3, SERPINF1, IGHG1 } \\
\text { C3, ANXA1, SERPINF1, SERPINA1, IGHG1 } \\
\text { C3, ANXA1, SERPINF1, SERPINA1, IGHG1 } \\
\text { C3, SERPINF1, IGHG1 } \\
\text { C3, SERPINF1, IGHG1 } \\
\text { C3, ANXA1, SERPINF1, S100A8 } \\
\text { ANXA1, SERPINA1, IGHG1 }\end{array}$ \\
\hline Tox list & $\begin{array}{l}\text { LXR/RXR activation } \\
\text { FXR/RXR activation } \\
\text { Positive acute phase response proteins }\end{array}$ & $\begin{array}{l}1.87 \mathrm{E}-11 \\
1.66 \mathrm{E}-09 \\
1.82 \mathrm{E}-08\end{array}$ & $\begin{array}{l}\text { S100A8, TF, C3, SERPINF1, SERPINA1, A1BG, ALB } \\
\text { TF, C3, SERPINF1, SERPINA1, AIBG, ALB } \\
\text { HP, C3, CP, SERPINA1 }\end{array}$ \\
\hline
\end{tabular}




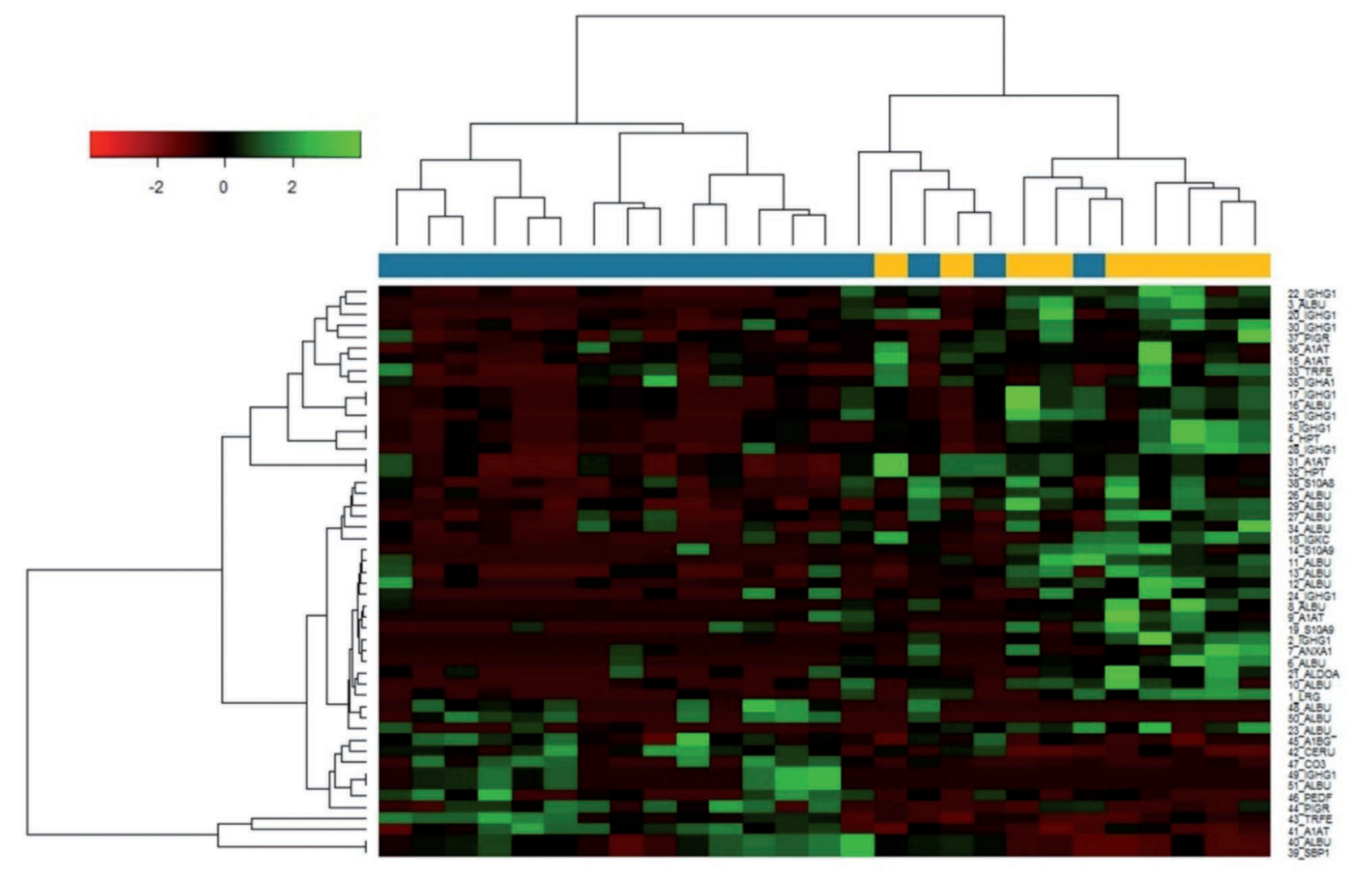

Figure 2. Heatmap and tree clustering. Heatmap and tree clustering of $\% \mathrm{~V}$ of differentially abundant protein spots detected by comparative analysis. AE and stable IPF groups are represented by yellow and light blue columns, respectively. The number of protein spots shown in Table $S 1$ is indicated on the rows.
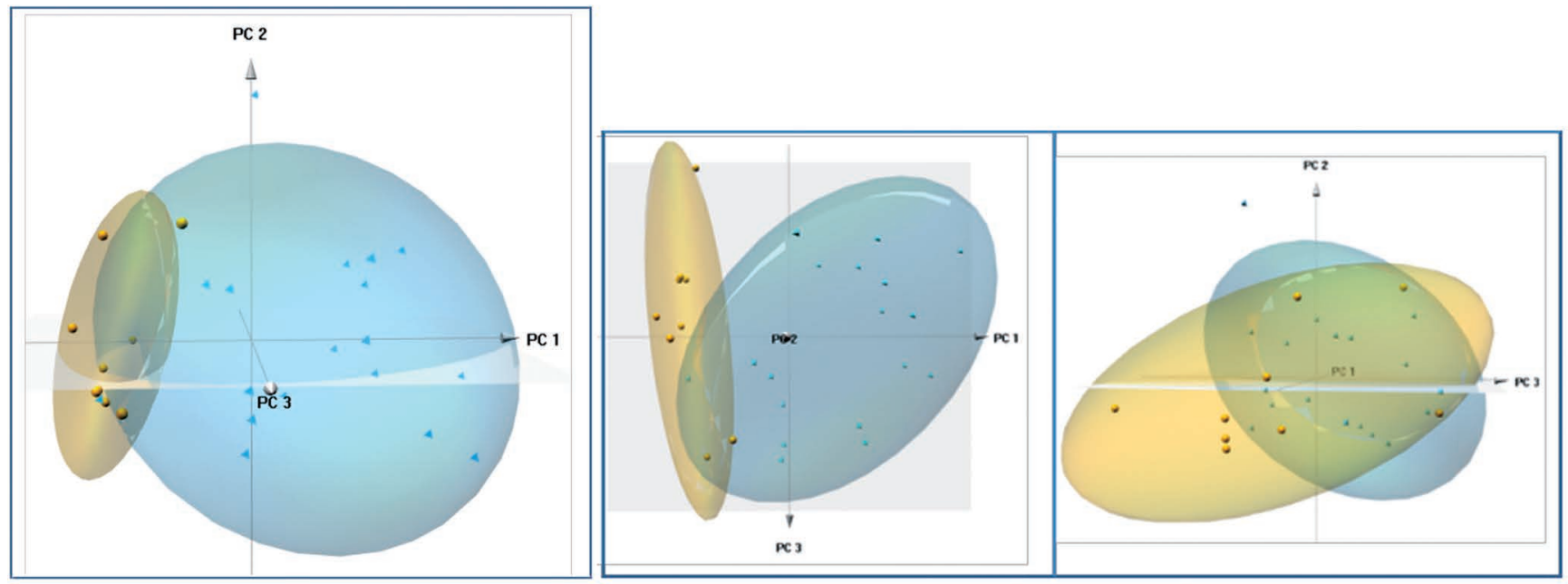

Figure 3. Principal Component Analysis. Three axonometric projections of 3D principal component analysis (PCA) plot obtained using normalized values of quantitatively differing protein spots in the two-dimensional electrophoresis comparison. AE and stable patients are indicated by yellow spheres and blue tetrahedrons, respectively. The ellipsoids enclose the 0.75 confidence interval for each group. 
targeted proteomic techniques. Setting our goal in pursuit of putative risk factors or proteins potentially involved in $\mathrm{AE}$ development, we established to collect the BAL Fluid samples at the starting manifestation of IPF symptoms and then to define the $\mathrm{AE}$ and Stable/Progressive groups after two years of clinical course monitoring. Even if the gender composition of overall cohort ( $\sim 62 \%$ male and $38 \%$ female) reflected the expected slightly higher male incidence of IPF, the AE group showed only one female case. We sifter through the potential disturbing effect of age and gender on the differentially protein abundances using general linear regression models.

\section{Conclusions}

Comparative proteomic analysis of BAL fluid samples from stable and AE IPF patients suggests a central role of macrophages and their fine regulation in AE development. Our results show the activation of receptors, such as LXR and FXR, involved in lipid metabolism, that different authors report to be particularly activated in foamy macrophages involved in atherosclerosis. Differentially identified proteins revealed a particular type of acute-phase response signaling as a potential cause of $\mathrm{AE}$
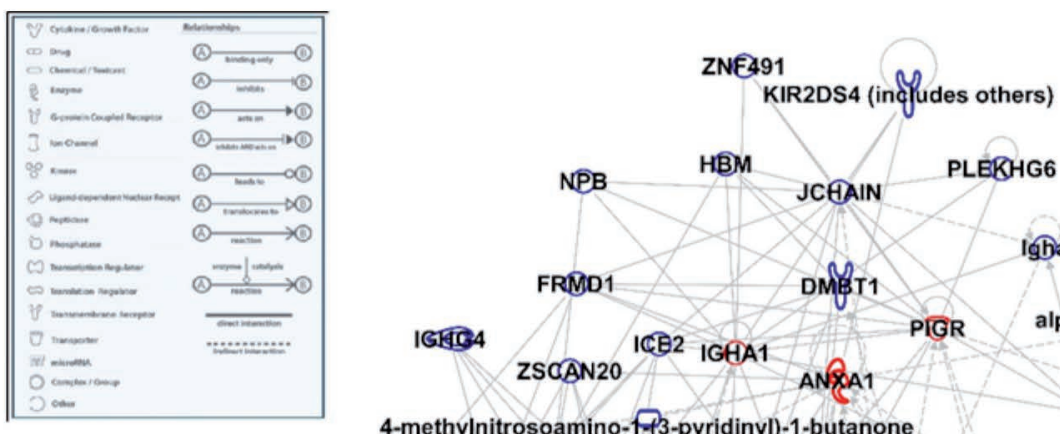

4-methylnitrosoamino-f-(3-pyridinyl)-1-butanone

chromium sivellestat IGBC benzo(a)pyrene D-galacfosamine

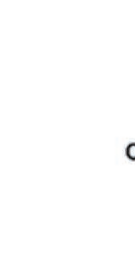
Ccoc82 19102

CEIARA

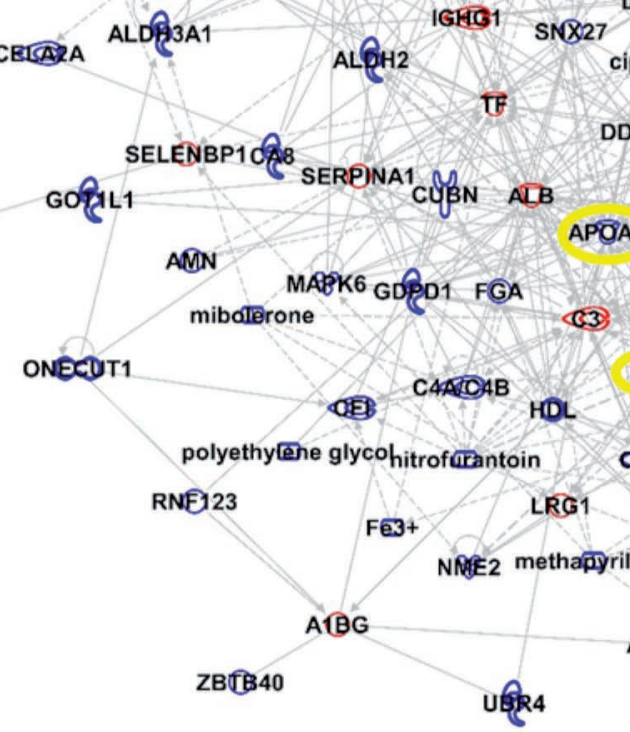
IGRO1 UGT1A7 (inctides others) KRT 9 ha-hydroxymyristic acid MMAि

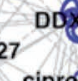
1 Igga $2 b$ ScoB $1 \mathrm{~A} 1$

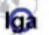
a

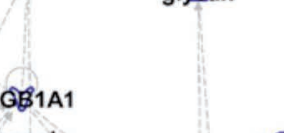

Spry 1 Orm1 (incl『es others) ACCC LOC61670 DDG Sâ3
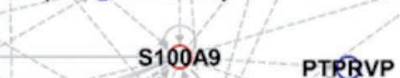
1670 TRBVP . 
involving A1AT C-36 peptide. Interestingly, our results indicate that proteins involved in clathrin-mediated endocytosis signaling, well known for the propagation of the fibrotic signaling $\beta$-catenin
WNT, may induce AE. Differential proteins found in the present study may also be involved in lung carcinogenesis and were substantially up regulated in AE IPF patients.
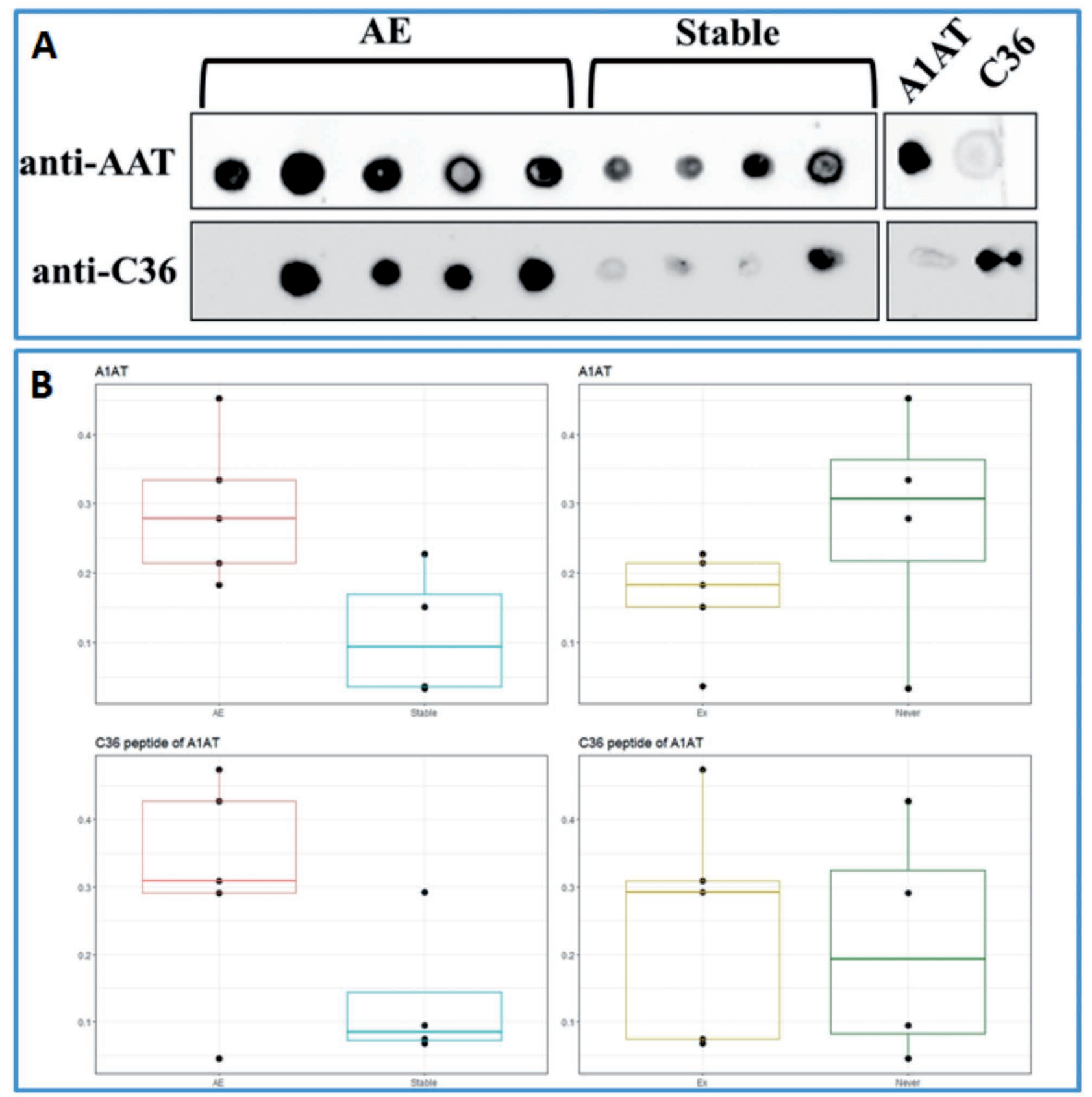

Figure 5. A) Dot blot of A1AT and C-36 peptide. BAL fluid protein abundances of $\alpha$-1-antitrypsin (A1AT) and its C-terminal fragment (C-36 peptide), corresponding to residues 359-394, were evaluated by dot-blot analysis in native condition; the lines A1AT and C-36 indicate the corresponding positive controls, which were commercial purified peptides. B) Box plots showing the Dot-blot intensity of A1AT and C36 peptide of A1AT according IPF clinical course (AE and stable patients in red and cyan, respectively) and according smoking status (never and ex-smokers in green and yellow, respectively). 


\section{References}

1. Nalysnyk L, Cid-Ruzafa J, Rotella P, Esser D. Incidence and prevalence of idiopathic pulmonary fibrosis: review of the literature. Eur Respir Rev 2012;21:355-61.

2. Raghu G, Collard HR, Egan JJ, et al. An official ATS/ERS/ JRS/ALAT statement: idiopathic pulmonary fibrosis: evidencebased guidelines for diagnosis and management. Am J Respir Crit Care Med 2011;183:788-824.

3. Balestrini JL, Chaudhry S, Sarrazy V, et al The mechanical memory of lung myofibroblasts. Integr Biol Quant Biosci Nano Macro 2012;4:410-21.

4. Jones MG, Fabre A, Schneider P, et al. Three-dimensional characterization of fibroblast foci in idiopathic pulmonary fibrosis. JCI Insight 2016;1. pii: e86375.

5. Pardo A, Cabrera S, Maldonado M, Selman M. Role of matrix metalloproteinases in the pathogenesis of idiopathic pulmonary fibrosis. Respir Res 2016;17:23.

6. Schilter H, Cantemir-Stone CZ, Leksa V, et al. The mannose-6phosphate analogue, PXS64, inhibits fibrosis via TGF- $\beta 1$ pathway in human lung fibroblasts. Immunol Lett 2015;165:90-101.

7. Cordier J-F, Cottin V. Neglected evidence in idiopathic pulmonary fibrosis: from history to earlier diagnosis. Eur Respir J 2013;42:916-23.

8. Vancheri C, Cottin V, Kreuter M, Hilberg O. IPF, comorbidities and management implications. Sarcoidosis Vasc Diffuse Lung Dis 2015;32:S17-23.

9. Poletti V, Ravaglia C, Buccioli M, et al. Idiopathic pulmonary fibrosis: diagnosis and prognostic evaluation. Respir Int Rev Thorac Dis 2013;86:5-12.

10. Fernández Pérez ER, Daniels CE, Schroeder DR, et al. Incidence, prevalence, and clinical course of idiopathic pulmonary fibrosis: a population-based study. Chest 2010;137:129-37.

11. Juarez MM, Chan AL, Norris AG, et al. Acute exacerbation of idiopathic pulmonary fibrosis-a review of current and novel pharmacotherapies. J Thorac Dis 2015;7:499-519.

12. Ryerson CJ, Cottin V, Brown KK, Collard HR. Acute exacerbation of idiopathic pulmonary fibrosis: shifting the paradigm. Eur Respir J 2015;46:512-20.

13. Kondoh Y, Cottin V, Brown KK. Recent lessons learned in the management of acute exacerbation of idiopathic pulmonary fibrosis. Eur Respir Rev 2017;26:170050.

14. Qiu M, Chen Y, Ye Q. Risk factors for acute exacerbation of idiopathic pulmonary fibrosis: A systematic review and metaanalysis. Clin Respir J 2018;12:1084-92.

15. Foster MW, Morrison LD, Todd JL, et al. Quantitative proteomics of bronchoalveolar lavage fluid in idiopathic pulmonary fibrosis. J Proteome Res 2015;14:1238-49.

16. Hara A, Sakamoto N, Ishimatsu Y. S100A9 in BALF is a candidate biomarker of idiopathic pulmonary fibrosis. Respir Med 2012;106:571-80.

17. Wietecha MS, Król MJ, Michalczyk ER, et al. Pigment epithelium-derived factor as a multifunctional regulator of wound healing. Am J Physiol Heart Circ Physio. 2015;309:H812-26.

18. Głodkowska-Mrówka E, Górska E, Ciurzyński M, et al. Proand antiangiogenic markers in patients with pulmonary complications of systemic scleroderma. Respir Physiol Neurobio. 2015;209:69-75.

19. Smadja DM, Nunes H, Juvin K, et al. Increase in both angiogenic and angiostatic mediators in patients with idiopathic pulmonary fibrosis. Pathol Biol (Paris) 2014;62:391-4.
20. Barratt SL, Flower VA, Pauling JD, Millar AB. VEGF (vascular endothelial growth factor) and fibrotic lung disease. Int J Mol Sci 2018;19:1269.

21. Agajanian MJ, Walker MP, Axtman AD, et al. WNT Activates the AAK1 kinase to promote clathrin-mediated endocytosis of LRP6 and establish a negative feedback loop. Cell Rep 2019;26:79-93.

22. Narayana YV, Gadgil C, Mote RD, et al. Clathrin-mediated endocytosis regulates a balance between opposing signals to maintain the pluripotent state of embryonic stem cells. Stem Cell Rep 2019;12:152-64.

23. Landi C, Bargagli E, Carleo A, Bianchi L, Gagliardi A, Prasse A, et al. A system biology study of BALF from patients affected by idiopathic pulmonary fibrosis (IPF) and healthy controls. Proteomics Clin Appl 2014;8:932-50.

24. Schupp JC, Binder H, Jäger B, Cillis G, Zissel G, MüllerQuernheim J, et al. Macrophage activation in acute exacerbation of idiopathic pulmonary fibrosis. PloS One 2015; 10:e116775.

25. Kan M, Shumyatcher M, Himes BE. Using omics approaches to understand pulmonary diseases. Respir Res 2017;18:149.

26. Collard HR, Ryerson CJ, Corte TJ, et al. Acute exacerbation of idiopathic pulmonary fibrosis. An International Working Group Report. Am J Respir Crit Care Med 2016;194:265-75.

27. Subramaniyam D, Glader P, von Wachenfeldt K, Bet al. C-36 peptide, a degradation product of alpha1-antitrypsin, modulates human monocyte activation through LPS signaling pathways. Int J Biochem Cell Biol 2006;38:563-75.

28. Donadio E, Piccolomini F, Dimuccio V, et al. Serum albumin fragmentation in end-stage renal disease patients--a pilot study. Clin Chem Lab Med 2009;47:1373-9.

29. Liang H, Hou H, Yi W, et al. Increased expression of pigment epithelium-derived factor in aged mesenchymal stem cells impairs their therapeutic efficacy for attenuating myocardial infarction injury. Eur Heart J 2013;34:1681-90.

30. Landi C, Bargagli E, Bianchi L, et al. Towards a functional proteomics approach to the comprehension of idiopathic pulmonary fibrosis, sarcoidosis, systemic sclerosis and pulmonary Langerhans cell histiocytosis. J Proteomics 2013;83:60-75.

31. Landi C, Bargagli E, Carleo A, et al. Bronchoalveolar lavage proteomic analysis in pulmonary fibrosis associated with systemic sclerosis: S100A6 and $14-3-3 \varepsilon$ as potential biomarkers. Rheumatol Oxf Engl 2019;58:165-78.

32. Thomas AC, Eijgelaar WJ, Daemen MJAP, Newby AC. The pro-fibrotic and anti-inflammatory foam cell macrophage paradox. Genomics Data 2015;6:136-8.

33. Thomas AC, Eijgelaar WJ, Daemen MJAP, Newby AC. Foam cell formation in vivo converts macrophages to a pro-fibrotic phenotype. PloS One 2015;10:e0128163.

34. Du S, Guan Z, Hao L, et al. Fructose-bisphosphate aldolase a is a potential metastasis-associated marker of lung squamous cell carcinoma and promotes lung cell tumorigenesis and migration. PloS One 2014;9:e85804.

35. Mittoo S, Gelber AC, Christopher-Stine L, et al. Ascertainment of collagen vascular disease in patients presenting with interstitial lung disease. Respir Med 2009;103:1152-8.

36. Liu Y, Luo X, Hu H, et al. Integrative proteomics and tissue microarray profiling indicate the association between overexpressed serum proteins and non-small cell lung cancer. PloS One 2012; 7:e51748.

37. Hong D, He G, Zhao S, t al. LRG1 modulates invasion and migration of glioma cell lines through TGF- $\beta$ signaling pathway. Acta Histochem 2015;117:551-8. 